\title{
Significance of ST-segment deviation in patients with acute pulmonary embolism and negative $T$ waves
}

\author{
Zhong-Qun Zhan ${ }^{1}$, Chong-Quan Wang ${ }^{2}$, Zhi-Xiao Wang ${ }^{2}$, Kjell C. Nikus ${ }^{3}$, Adrian Baranchuk ${ }^{4}$, \\ Rui-Xia Yuan ${ }^{2}$, Daniel D. Anselm${ }^{4}$, Andrés Ricardo Pérez-Riera ${ }^{5}$, Bo Yang ${ }^{1}$ \\ ${ }^{1}$ Renmin Hospital of Wuhan University, China \\ ${ }^{2}$ Shiyan Taihe Hospital, Hubei University of Medicine, China \\ ${ }^{3}$ Tampere University Hospital, and School of Medicine, Tampere University, Finland \\ ${ }^{4}$ Kingston General Hospital, Queen's University, Canada \\ ${ }^{5} \mathrm{ABC}$ Faculty of Medicine, ABC Foundation, Brazil
}

\begin{abstract}
Background: Common electrocardiogram (ECG) manifestations in acute pulmonary embolism (APE) include ST-segment deviation (STDV) along with negative T-waves (NTW). STDV could occur in 3 typical ischemic patterns: (i) the left ventricular (LV) subendocardial ischemic pattern; (ii) the right ventricular (RV) transmural ischemic pattern; and (iii) the LV subendocardial plus RV transmural ischemic pattern. The purpose of this study was to evaluate the relationship of STDV and adverse clinical outcomes and to identify the relationship of relatively normal ECG and favorable clinical outcomes.
\end{abstract}

Methods: Retrospective analysis of electronic charts in APE patients was performed in a tertiary hospital. ECGs on admission were obtained and classified as with or without STDV. Adverse clinical outcomes were defined as need to intensify therapy and 30-day mortality. Relatively normal ECG was defined as without any STDV, abnormal QRS morphology in lead V1 and S1Q3T3.

Results: From a total of 210 patients with NTW, 131 had STDV $\geq 0.1 \mathrm{mV}$, while 79 did not. Patients with STDV had worse evolution: higher incidence of dyspnea, hypotension, cardiogen$i c$ shock, intensification of therapy, and death compared to patients without $S T D V(p=0.001$ for each variable). The majority (89\%) of the patients with STDV presented with 1 of the 3 typical ischemic ECG patterns. LV subendocardial ischemic pattern $(O R=4.963, p=0.004)$, $R V$ transmural ischemic pattern $(O R=3.128, p=0.021)$ and $L V$ subendocardial plus $R V$ transmural ischemic pattern $(O R=3.036, p=0.017)$ independently predicted the need to intensify therapy. $R V$ transmural ischemic pattern $(O R=4.227, p=0.031)$ and LV subendocardial plus $R V$ transmural ischemic pattern $(O R=4.022, p=0.032)$ independently predicted 30-day mortality. Compared to the patients with abnormal ECG, the patients with relatively normal ECG had a significant lower incidence of death (0\% vs. 16\%; $p=0.001$ ) and need to intensify therapy during hospitalization ( $6 \%$ vs. 30\%; $p=0.002$ ).

Conclusions: Ischemic ECG patterns are common ECG manifestations of APE and predict worse evolution and 30-day mortality. Additionally, relatively normal ECGs may associate with favorable clinical outcomes. (Cardiol J 2015; 22, 5: 583-589)

Key words: acute pulmonary embolism, electrocardiogram, ST-segment deviation

Address for correspondence: Bo Yang, MD, Department of Cardiology, Renmin Hospital of Wuhan University, Wuhan, Hubei Province, China, e-mail: 2622915280@qq.com

Received: 12.03.2015 Accepted: 04.04.2015 


\section{Introduction}

Negative T waves (NTW) in the inferior and precordial leads is a recognized electrocardiogram (ECG) manifestation of acute pulmonary embolism (APE) [1, 2] and has been suggested as a marker of right ventricular (RV) strain (RVS) [3] or RV dysfunction (RVD) $[4,5]$.

ST-segment deviation (STDV) (elevation and/ /or depression) is another common ECG manifestation of APE [6-8]. STDV could occur in 3 specific ischemic patterns: (i) left ventricular (LV) subendocardial ischemic pattern; (ii) RV transmural ischemic pattern; and (iii) LV subendocardial plus $\mathrm{RV}$ transmural ischemic pattern [9].

The purpose of this study was to evaluate the relationship of STDV and adverse clinical outcomes in APE patients with NTW and to investigate the relationship between relatively normal ECG and favorable clinical outcomes.

\section{Methods}

\section{Patient population}

There were 342 patients diagnosed with APE in our hospital from January 2008 to July 2014, including 315 patients confirmed by multidetector computed tomographic (MDCT) pulmonary angiography and 27 patients highly suspected by ventilation-perfusion scintigraphy. Using a retrospective analysis, 210 patients with NTW were included in this study: 131 patients with and 79 patients without STDV $\geq 0.1 \mathrm{mV}$ (Fig. 1).

The inclusion criteria were: (i) acute onset of clinical signs and symptoms before admission suggesting APE; (ii) APE confirmed by MDCT pulmonary angiography; and (iii) admission ECG with NTW $\geq 0.1 \mathrm{mV}$ in at least 2 leads out of the inferior (II, III and aVF) and V1-V4 leads.

The exclusion criteria were: (i) history of chronic heart failure or confirmed pulmonary hypertension associated with other cardiopulmonary disease (chronic obstructive pulmonary disease was not excluded if there was no confirmed history of pulmonary hypertension by echocardiogram); (ii) concomitant cardiac disease possibly causing NTW in the inferior or precordial leads, such as acute coronary syndrome, hypertrophic cardiomyopathy or tako-tsubo cardiomyopathy confirmed by coronary and/or LV angiography; (iii) ECG manifestation of complete left branch bundle block, LV hypertrophy or ventricular pacing.

The study was approved by the Shiyan Taihe Hospital bioethical committee.

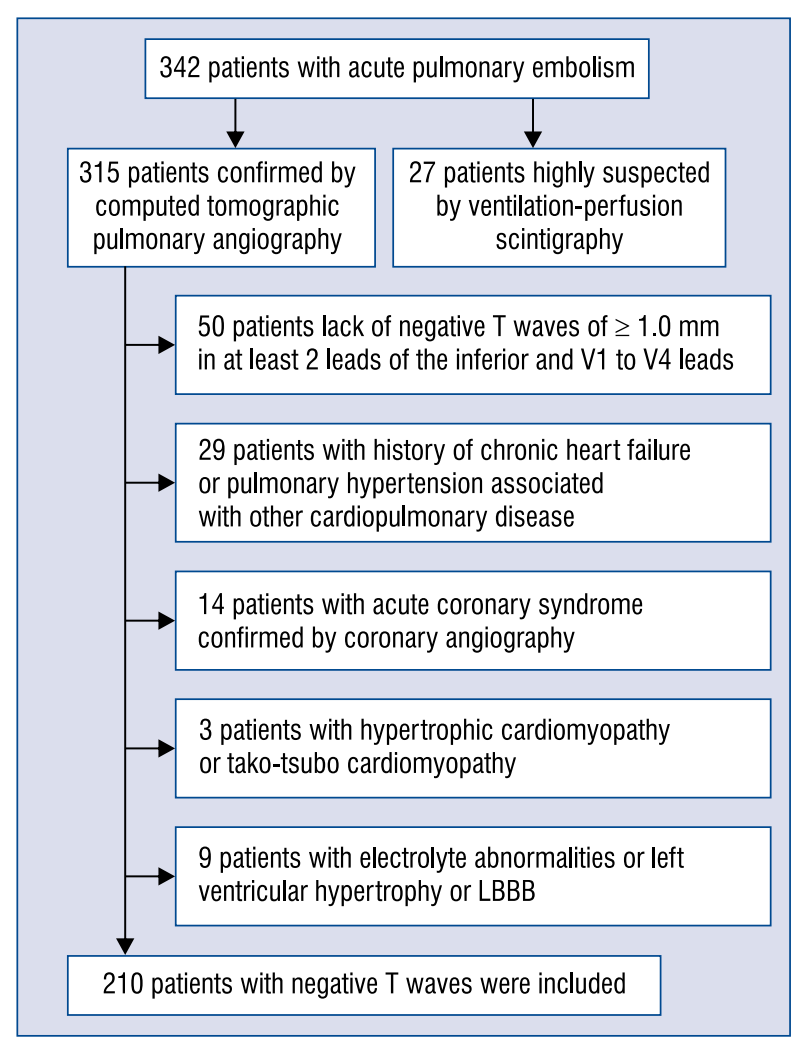

Figure 1. Flow chart of patient selection with acute pulmonary embolism; LBBB - left bundle branch block.

\section{Electrocardiogram}

After admission, an ECG using a paper speed of $25 \mathrm{~mm} / \mathrm{s}$ with standardization of $1 \mathrm{mV} / 10 \mathrm{~mm}$ was recorded. Both ST-segment elevation (STE) and ST-segment depression (STD) were measured at the J-point in all leads and were considered present if the J-point was $\geq 0.1 \mathrm{mV}$ above or below the isoelectric line. The LV subendocardial ischemic pattern was defined as STE in lead aVR with concomitant STD in leads V4/V5 to V6 (Fig. 2A). The $\mathrm{RV}$ transmural ischemic pattern was defined as STE in at least 2 leads of the leads V1 to V3 (Fig. 2B). The LV subendocardial plus RV transmural ischemic pattern was defined as STE in leads III and/ /or V1/V2 with concomitant STD in leads V4/V5 to V6 (Fig. 2C). The other abnormal ECG criteria associated with APE are identical to our previous study [9]. Relatively normal ECG was defined as an ECG without any STDV, abnormal QRS morphology in lead V1 (notched S wave, right bundle branch block [RBBB] or QR sign) and S1Q3T3. Abnormal ECG was defined as the presence of at least one of the STDV, abnormal QRS morphology in lead V1 and S1Q3T3. Patients were categorized into two groups (with or without STDV) based on the ECG 


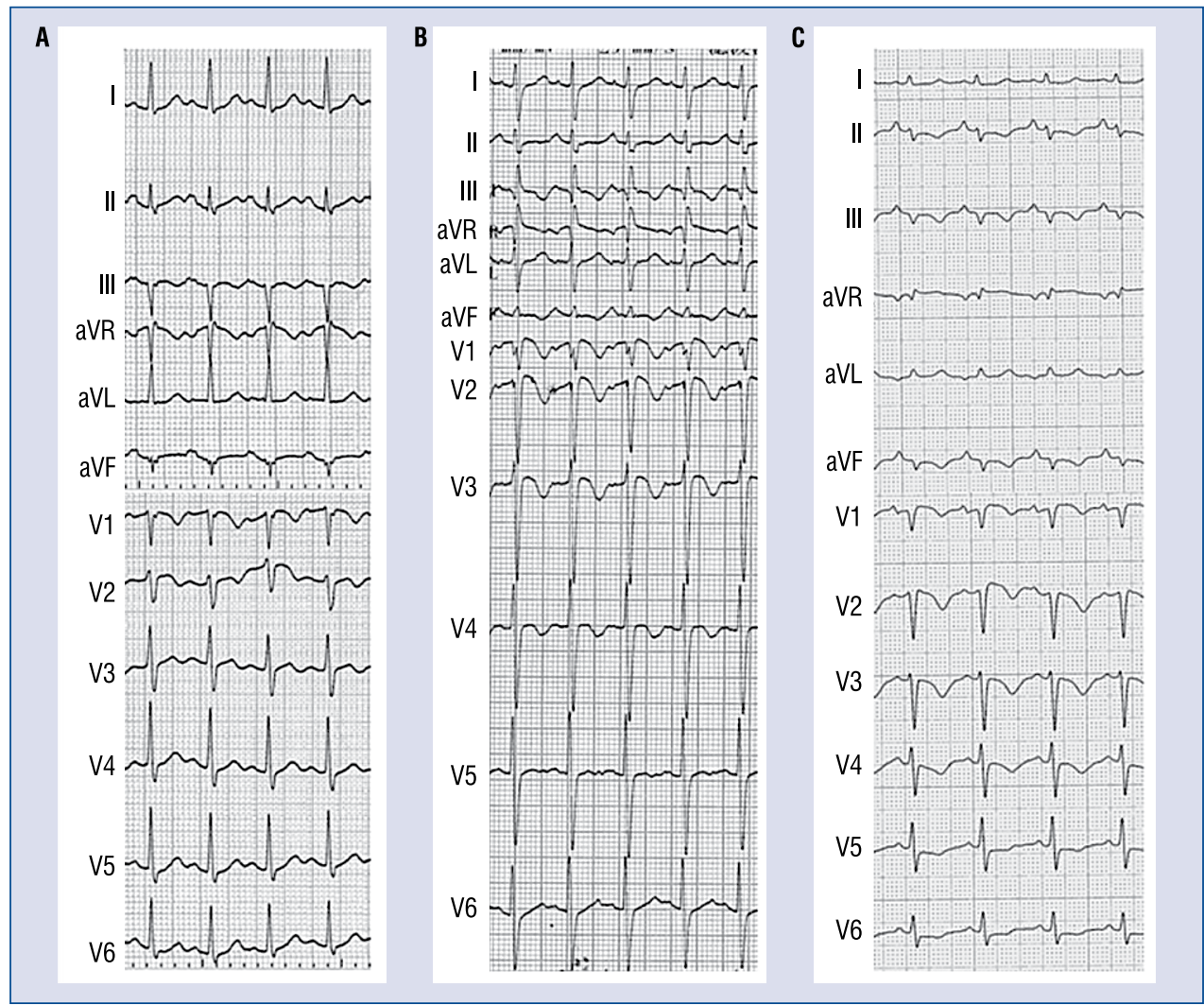

Figure 2. Examples of the ischemic electrocardiographic patterns. A. Left ventricular (LV) subendocardial ischemic pattern; B. Right ventricular (RV) transmural ischemic pattern; C. LV plus RV ischemic pattern.

findings. All ECGs were evaluated separately by 2 independent investigators without knowledge of the patients' clinical condition. Disagreement between the 2 investigators was resolved by consensus.

\section{Adverse clinical outcomes}

The following adverse clinical outcomes were recorded: need to intensify therapy based on the Management Strategies and Prognosis in Pulmonary Embolism Trial-3 (MAPPET-3) study criteria [10], including cardiac arrest, need for inotropic support, mechanical ventilation for respiratory support and treatment with thrombolysis or surgical embolectomy; and 30-day mortality.

\section{Statistical analysis}

All data were analyzed by SPSS 12.0. Data were expressed as mean \pm standard deviation for continuous variables and rates (\%) for categorical variables. The Student's U test was used for comparison of continuous variables. Categorical variables were compared using the $\chi^{2}$ test or the Fisher exact test. All ECG variables were analyzed by multivariate logistic regression to identify predictors of the need to intensify therapy and 30-day mortality. Odds ratios (ORs) and 95\% confidence intervals (CIs) were calculated. A 2 -tailed probability value $<0.05$ was considered statistically significant.

\section{Results}

Among the 210 patients, 185 (88\%) patients presented with NTW in at least 1 inferior and 1 precordial lead. Of the 131 patients with STDV, frequent findings were STE in leads V1 (47\%), V2 (31\%), III (28\%) and aVR (68\%) and STD in leads I (60\%), and V4/V5-V6 (60\%). There were 24 (18\%) patients with the LV subendocardial ischemic pattern, $41(31 \%)$ patients with the RV transmural ischemic pattern, and $52(40 \%)$ with the LV subendocardial plus RV transmural ischemic pattern. The remaining $14(11 \%)$ patients did not meet the criteria of the pre-specified three ischemic ECG patterns. The distribution of STDV is listed in Table 1.

The two groups were not statistically different with regard to gender, age, clinical symptoms of hemoptysis, chest discomfort, cough or syncope. 
Table 1. Distribution of ST-segment deviation in patients with ST-segment deviation $(n=131)$.

\begin{tabular}{lc} 
STE in V1 $\geq 0.1 \mathrm{mV}$ & $61(47 \%)$ \\
STE in $\mathrm{V} 2 \geq 0.1 \mathrm{mV}$ & $41(31 \%)$ \\
STE in $\mathrm{V} 3 \geq 0.1 \mathrm{mV}$ & $11(8 \%)$ \\
STE in III $\geq 0.1 \mathrm{mV}$ & $37(28 \%)$ \\
STE in III and aVF $\geq 0.1 \mathrm{mV}$ & $14(11 \%)$ \\
STE in aVR $\geq 0.1 \mathrm{mV}$ & $89(68 \%)$ \\
STD in I $\geq 0.1 \mathrm{mV}$ & $78(60 \%)$ \\
STD in V4/V5-V6 $\geq 0.1 \mathrm{mV}$ & $78(60 \%)$ \\
LV subendocardial ischemic pattern & $24(18 \%)$ \\
RV transmural ischemic pattern & $41(31 \%)$ \\
LV plus RV transmural ischemia pattern & $52(40 \%)$ \\
\hline
\end{tabular}

LV — left ventricle; RV — right ventricle; STE — ST-segment elevation; STD - ST-segment depression

Comparing to patients without STDV, patients with STDV had a shorter time from symptom onset to admission (6.6 \pm 3.2 vs. $9.2 \pm 4.8$ day, $\mathrm{p}=0.004)$; lower systolic $(117 \pm 29$ vs. $130 \pm 25 \mathrm{~mm} \mathrm{Hg}$, $\mathrm{p}=0.001)$ and diastolic blood pressure $(74 \pm 22$ vs. $81 \pm 14 \mathrm{~mm} \mathrm{Hg}, \mathrm{p}=0.004)$; higher incidence of dyspnea on exertion ( $84 \%$ vs. $65 \%, \mathrm{p}=0.001)$ and at rest ( $45 \%$ vs. $22 \%, \mathrm{p}=0.001)$; hypotension
(17\% vs. $0 \%, \mathrm{p}=0.001)$; cardiogenic shock $(27 \%$ vs. $5 \%, \mathrm{p}=0.001)$; death $(18 \%$ vs. $5 \%, \mathrm{p}=0.006)$; and the need to intensify therapy (35\% vs. $11 \%$, $\mathrm{p}=0.001)$. Demographic and clinical data are listed in Table 2.

The multivariate logistic regression analysis of the ECG parameters for the prediction of 30-day mortality and the need to intensify therapy during hospitalization are listed in Table 3 . We found that the LV subendocardial ischemic pattern (OR $=4.963, \mathrm{p}=0.004)$, the RV transmural ischemic pattern $(\mathrm{OR}=3.128, \mathrm{p}=0.021)$ and the LV subendocardial plus RV transmural ischemic pattern $(\mathrm{OR}=3.036, \mathrm{p}=0.017)$ independently predicted the need to intensify therapy during hospitalization. In addition, the RV transmural ischemic pattern $(\mathrm{OR}=4.227, \mathrm{p}=0.031)$ and the $\mathrm{LV}$ subendocardial plus RV transmural ischemic pattern $(\mathrm{OR}=4.022$, $\mathrm{p}=0.032$ ) independently predicted 30 -day mortality.

Of the 36 patients fulfilling the criteria of relatively normal ECG on admission, there were 2 patients needing to intensify therapy and no death. Accordingly, compared to the patients with abnormal ECG, the patients with relatively normal ECG had a significant lower incidence of death $(0 \%$ vs. $16 \%, p=0.001$ ) and need to intensify therapy during hospitalization ( $6 \%$ vs. $30 \%, \mathrm{p}=0.002)$.

Table 2. Demographic and clinical data.

\begin{tabular}{lccc}
\hline & $\begin{array}{c}\text { APE patients with } \\
\text { ST deviation (n= 131) }\end{array}$ & $\begin{array}{c}\text { APE patients without } \\
\text { ST deviation (n= 79) }\end{array}$ & P \\
\hline Gender (male/female) & $55 / 76$ & $37 / 42$ & $\mathrm{NS}$ \\
Age [years] & $59 \pm 14$ & $56 \pm 15$ & $\mathrm{NS}$ \\
Time from symptom onset to admission [day] & $6.6 \pm 3.2$ & $9.2 \pm 4.8$ & 0.004 \\
Heart rate [bpm] & $110 \pm 23$ & $95 \pm 23$ & 0.001 \\
Systolic BP [mm Hg] & $117 \pm 29$ & $130 \pm 25$ & 0.001 \\
Diastolic BP [mm Hg] & $74 \pm 22$ & $81 \pm 14$ & 0.004 \\
Symptoms on admission: & & $7(9 \%)$ & $\mathrm{NS}$ \\
Hemoptysis & $8(6 \%)$ & $51(65 \%)$ & 0.001 \\
Dyspnea on exertion & $110(84 \%)$ & $17(22 \%)$ & 0.001 \\
Dyspnea on rest & $59(45 \%)$ & $30(38 \%)$ & $\mathrm{NS}$ \\
Chest discomfort & $51(39 \%)$ & $19(24 \%)$ & $\mathrm{NS}$ \\
Cough & $26(20 \%)$ & $19(24 \%)$ & $\mathrm{NS}$ \\
Syncope & $38(29 \%)$ & $0(0 \%)$ & 0.001 \\
Hypotension & $22(17 \%)$ & $4(5 \%)$ & 0.001 \\
Cardiogenic shock & $36(27 \%)$ & $4(5 \%)$ & 0.006 \\
30-day death & $24(18 \%)$ & $9(11 \%)$ & 0.001 \\
Need to intensify therapy & $46(35 \%)$ & & \\
\hline
\end{tabular}

Data are expressed as mean \pm standard deviation for continuous variables and as number (\%) or ratio for categorical variables; APE — acute pulmonary embolism; BP — blood pressure; NS — not significant 
Table 3. Multivariate logistic regression analysis of electrocardiogram (ECG) parameters for the prediction of 30-day mortality and the need to intensify therapy during hospitalization.

\begin{tabular}{|c|c|c|c|c|c|c|}
\hline \multirow[t]{2}{*}{ ECG parameter } & \multicolumn{2}{|c|}{$\begin{array}{l}\text { 30-day death } \\
(n=28)\end{array}$} & \multirow[t]{2}{*}{$\mathbf{P}$} & \multicolumn{2}{|c|}{$\begin{array}{l}\text { Need to intensify } \\
\text { therapy }(n=55)\end{array}$} & \multirow[t]{2}{*}{$\mathbf{P}$} \\
\hline & OR & Cl & & OR & $\mathrm{Cl}$ & \\
\hline Atrial fibrillation/atrial flutter & 1.360 & $0.386-4.795$ & NS & 1.022 & $0.354-2.946$ & NS \\
\hline S103T3 & 3.169 & $0.988-10.169$ & 0.052 & 1.792 & $0.787-4.082$ & NS \\
\hline Left axis deviation & 1.266 & $0.435-3.683$ & NS & 0.566 & $0.233-1.372$ & NS \\
\hline Right axis deviation & 1.022 & $0.340-3.072$ & NS & 1.061 & $0.441-2.553$ & NS \\
\hline Low QRS voltage & 1.633 & $0.429-6.219$ & NS & 1.003 & $0.36-3.086$ & NS \\
\hline Clockwise rotation & 0.287 & $0.052-1.584$ & NS & 0.532 & $0.162-1.746$ & NS \\
\hline Notched S wave in V1 & 1.225 & $0.397-3.778$ & NS & 1.535 & $0.599-3.938$ & NS \\
\hline RBBB in V1 & 0.643 & $0.162-2.549$ & NS & 0.916 & $0.329-2.553$ & NS \\
\hline Qr sign in V1 & 0.778 & $0.239-2.530$ & NS & 1.036 & $0.413-2.598$ & NS \\
\hline Number of leads with negative $T$ waves & 1.181 & $0.960-1.454$ & NS & 1.098 & $0.928-1.299$ & NS \\
\hline LV subendocardial ischemic pattern & 3.711 & $0.781-17.628$ & NS & 4.963 & $1.668-14.768$ & 0.004 \\
\hline $\mathrm{RV}$ transmural ischemic pattern & 4.227 & $1.144-15.625$ & 0.031 & 3.128 & $1.190-8.225$ & 0.021 \\
\hline LV plus RV transmural ischemia pattern & 4.022 & $1.130-14.319$ & 0.032 & 3.036 & $1.219-7.562$ & 0.017 \\
\hline
\end{tabular}

$\mathrm{Cl}$ — confidence interval; LV — left ventricle; NS — not significant; OR — odds ratio; RBBB — right bundle branch block; RV — right ventricle

\section{Discussion}

STDV is considered a common, but non-specific, ECG manifestation in patients with APE by some authors $[11,12]$. However, the literature suggests that the distribution of STDV has consistent regularity and mechanisms [6-9, 13-16]. STE in leads V1, III, aVR and STD in leads I and V4/ V5-V6 are frequently observed in APE $[6,7,9]$. In our study, the most common STDV were also STE in leads V1, V2, III and aVR and STD in leads I and V4/V5-V6.

We have described that 3 distinct ischemic ECG patterns represent different manifestations of RV and LV transmural and subendocardial ischemia [9]. According to our results, these ECG patterns are frequent (89\%) in APE patients with STDV and NTW. The mechanism of the ischemic patters has been attributed to hypotension, hypoxemia, pulmonary artery hypertension, and catecholamine surge $[9,14]$.

In this study, we have demonstrated that STDV with concomitant NTW had worse evolution compared to NTW without STDV. Patients with STDV had a higher incidence of dyspnea on exertion or on rest, hypotension, cardiogenic shock, need to intensify the therapy, and higher 30-day mortality compared to patients without STDV. The 3 distinct ischemic ECG patterns could be used to identify patients at higher risk of worse clinical adverse outcomes. To our knowledge, we are the first to demonstrate that the relatively normal ECGs (no STDV, absence of notched S wave or RBBB or QR sign in lead V1 and S1Q3T3) are associated with favorable clinical outcomes. It should be pointed out that the relatively normal ECG still present with NTW $\geq 0.1 \mathrm{mV}$ in at least 2 leads out of the inferior (II, III and aVF) and V1-V4 leads.

NTW in the inferior and precordial leads has been suggested as a marker of RVS [3] or RVD $[4,5]$. However, another study [17] suggested that an increase in the amplitude of NTW in the precordial leads after thrombolytic therapy reflects an improvement in cardiopulmonary hemodynamics with concomitant decrease of both RV diameter and mean pulmonary artery pressure. We previously described the dynamic ECG changes in 2 patients and found that NTW developed later than STE and that the NTW persisted beyond recovery of RVD [14]. In another study [9], none of the 8 patients with acquired APE during hospitalization presented with NTW in precordial leads during hypotension or cardiogenic shock; additionally, all 8 patients showed STE and/or STD. Hence, NTW in the inferior and precordial leads without STDV might represent a "post-ischemic" ECG stage, thus reflecting a previous, transient, stage of RV stretch and ischemia $[9,14]$. We also speculate 
that more leads with NTW reflect more extensive prior ischemia. Hence, there is a conflicting relationship between NTW and RVD or the severity of the APE in the literature. Association of NTW and RVD or the severity of the APE can be found in patients with STDV [14] and in patients who were not treated for APE $[3,5]$. More leads with NTW are also related to worse short-term prognosis $[18,19]$. However, there is a poor relationship in patients after effective treatment where the RVD has possibly been recovered $[14,17]$.

The following are commonalities between the findings of our groups' studies and the studies by the Kukla's group [6, 7, 19]. First, STDV reflects worse prognosis compared to NTW. Second, STE in leads V1, III and aVR and STD in leads V4/V5-V6 and NTW in the inferior and precordial leads are common ECG manifestations of APE. However, the differences between our study and Kukla's group are obvious. We identified three types of ischemic ECG patterns that are common ECG manifestations and of prognostic significance. Kukla et al. [7] found that "global ischemic pattern" (presence of at least one lead with STE in leads III, aVR and V1-V4 and at least two leads with STD in I, aVL and V4-V6) similar to our LV subendocardial plus $\mathrm{RV}$ transmural ischemic pattern was not associated with worse outcome in intermediate-risk APE patients. However, "ST-segment ischemic pattern" (presence of at least one lead with STE in leads III, aVR and V1-V4 or at least two leads with STD in leads I, aVL and V4-V6) is associated with a worse prognosis in intermediate-risk APE patients. We suggest that this difference is mainly attributed to having a lower incidence of global ischemic pattern than that of ST-segment ischemic pattern (13.9\% vs. $44.5 \%$ ) in intermediate-risk APE patients. Incidentally, the LV subendocardial plus RV transmural ischemic pattern is the main ECG presentation $(80 \%)$ in the high-risk APE patients [9] and signifies worse adverse clinical outcomes in this study. According to Kukla et al. [19], the mortality rate was significantly higher in patients with NTW in the inferior and precordial leads compared to the group without NTW. In addition, as compared to those with NTW in $<5$ leads, patients with NTW $\geq 5$ leads experienced significantly higher rates of death and complications. We did not find the number of leads with NTW to be associated with worse adverse clinical outcomes. This phenomenon may be attributed to a smaller number of patients in our study (210 vs. 470 patients) and methodological differences - we used the number of leads with NTW, while Kukla et al. [19] used a cut-off of 5 leads in their analyses. In addition, our group used J-point as the measuring point of STDV while the Kukla's group used $80 \mathrm{~ms}$ after the J-point; this may account for further discrepancy between our studies.

\section{Limitations of the study}

Our study has several limitations. It is a retrospective single center study. Different departments and different physicians may have variable criteria with regards to need to intensify therapy. There are many factors influencing STDV and we could not rule out all the confounding factors such as concomitant coronary artery disease or paradoxical coronary embolism.

\section{Conclusions}

Ischemic ECG patterns are common ECG manifestations of APE. They are clinically important as they could predict adverse clinical outcomes. Prompt recognition of these patterns may help in avoiding inaccurate diagnosis leading to unnecessary medical investigations and treatments and may aid in detecting high-risk patients with APE. Relatively normal ECGs may associate with favorable clinical outcomes.

\section{Conflict of interests: None declared}

\section{References}

1. Kosuge M, Ebina T, Hibi K et al. Differences in negative T waves among acute coronary syndrome, acute pulmonary embolism, and Takotsubo cardiomyopathy. Eur Heart J, 2012; 1: 349-357. doi: $10.1177 / 2048872612466790$.

2. Jankowski K, Kostrubiec M, Ozdowska P et al. Electrocardiographic differentiation between acute pulmonary embolism and non-ST elevation acute coronary syndromes at the bedside. Ann Noninvasive Electrocardiol, 2010; 15: 145-150. doi: 10.1177/2048872612466790.

3. Vanni S, Polidori G, Vergara R, et al. Prognostic value of ECG among patients with acute pulmonary embolism and normal blood pressure. Am J Med, 2009; 122: 257-264. doi: 10.1016/j. amjmed.2008.08.031.

4. Choi BY, Park DG. Normalization of negative T-wave on electrocardiography and right ventricular dysfunction in patients with an acute pulmonary embolism. Korean J Intern Med, 2012; 27: 53-59. doi: 10.3904/kjim.2012.27.1.53.

5. Kim SE, Park DG, Choi HH et al. The best predictor for right ventricular dysfunction in acute pulmonary embolism: comparison between electrocardiography and biomarkers. Korean Circ J, 2009; 39: 378-381. doi: 10.4070/kcj.2009.39.9.378.

6. Kukla P, McIntyre WF, Fijorek K et al. Electrocardiographic abnormalities in patients with acute pulmonary embolism complicated by cardiogenic shock. Am J Emerg Med, 2014; 32: 507-510. doi: 10.1016/j.ajem.2014.01.043.

7. Kukla P, McIntyre WF, Fijorek K et al. Use of ischemic ECG patterns for risk stratification in intermediate-risk patients with 
acute PE. Am J Emerg Med, 2014; 32: 1248-1252. doi: 10.1016/j. ajem.2014.07.029.

8. Janata K, Hochtl T, Wenzel C et al. The role of ST-segment elevation in lead aVR in the risk assessment of patients with acute pulmonary embolism. Clin Res Cardiol, 2012; 101: 329-337. doi: 10.1007/s00392-011-0395-z.

9. Zhan $Z Q$, Wang $\mathrm{CQ}$, Nikus $\mathrm{KC}$ et al. Electrocardiogram Patterns during Hemodynamic Instability in Patients with Acute Pulmonary Embolism. Ann Noninvasive Electrocardiol, 2014; 19: 543-551. doi: 10.1111/anec.12163.

10. Konstantinides S, Geibel A, Heusel G et al. Heparin plus alteplase compared with heparin alone in patients with submassive pulmonary embolism. N Engl J Med, 2002; 347: 1143-1150 .

11. Stein PD, Terrin ML, Hales CA et al. Clinical, laboratory, roentgenographic, and electrocardiographic findings in patients with acute pulmonary embolism and no pre-existing cardiac or pulmonary disease. Chest, 1991; 100: 598-603.

12. Agrawal N, Ramegowda RT, Patra S et al. Predictors of inhospital prognosis in acute pulmonary embolism: keeping it simple and effective. Blood Coagul Fibrinolysis, 2014; 25: 492-500. doi: 10.1097/MBC.0000000000000093.
13. Geibel A, Zehender M, Kasper W et al. Prognostic value of the ECG on admission in patients with acute major pulmonary embolism. Eur Respir J, 2005. 25: 843-848.

14. Zhong-Qun Z, Bo Y, Nikus KC et al. Correlation between ST-segment elevation and negative $T$ waves in the precordial leads in acute pulmonary embolism: insights into serial electrocardiogram changes. Ann Noninvasive Electrocardiol, 2014; 19: 398-405. doi: 10.1111/anec.12115.

15. Zhan $Z Q$, Wang $C Q$, Nikus $\mathrm{KC}$ et al. Brugada phenocopy in acute pulmonary embolism. Int J Cardiol, 2014; 177: e153-5. doi: 10.1016/j.ijcard.2014.09.046.

16. Zhan ZQ, Wang CQ, Baranchuk A. Acute pulmonary embolism with ST-segment elevation in the inferior leads. Int J Cardiol, 2014; 177: 718-719.

17. Yoshinaga $\mathrm{T}$, Ikeda $\mathrm{S}$, Nishimura $\mathrm{E}$ et al. Serial changes in negative $\mathrm{T}$ wave on electrocardiogram in acute pulmonary thromboembolism. Int J Cardiol, 1999 ; 72: 65-72.

18. Kosuge M, Kimura K, Ishikawa T et al. Prognostic significance of inverted $\mathrm{T}$ waves in patients with acute pulmonary embolism. Circ J, 2006; 70: 750-755.

19. Kukla P, McIntyre WF, Fijorek $\mathrm{K}$ et al. T-wave inversion in patients with acute pulmonary embolism: Prognostic value. Heart Lung, 2015; 44: 68-71. doi: 10.1016/j.hrtlng.2014.10.003. 